\title{
THE ROLE OF A FUZZY PID ON A MECHATRONICS CONTROL SYSTEM OF AN INDUSTRIAL ROBOT
}

\author{
Yanbo Ben \\ School of Electronic and Electrical Engineering, Zibo Vocational Institute, Zibo, 255000, China \\ Email: benyanbosdzb@126.com
}

\begin{abstract}
The purpose is to integrate the fuzzy controller with the traditional PID (Proportion Integral Deferential) controller and apply it to the mechatronics control of industrial robots. Here, the fuzzy controller and the traditional PID controller are innovatively combined based on the working principle of fuzzy controller and traditional PID control. Consequently, the fuzzy PID controller is introduced, and the calculation process is analysed. Then, the mechatronics control system is proposed for the industrial robot and simulated through an industrial transport robot. The results show that the industrial transport robot has a fast response to the grasping operation, but the speed fluctuation is large. The system has an overshoot and a small steady-state error, but the error conforms to the standardized design established by Mamdani. When the robot moves forward and backward, it will move around the set value and stop moving until the system reaches stability. The fluctuation of the system response is small. The speed change value in 10 seconds is between $[-0.02,0.01]$, and there is no significant oscillation. Meanwhile, the response time of the system is short during operation. This shows that the mechatronics control system of industrial robots based on fuzzy PID has strong stability, controlling the transport robot to respond timely and perform stably.
\end{abstract}

Keywords: Fuzzy PID Controller; Industrial Transport Robot; Mechatronics Control System; Stability.

\section{Introduction}

Industrial robots are emerging industries in the information age. It provides extremely important technical support for the current industrial automation and advanced manufacturing. It plays an increasingly important role in expanding enterprise production and promoting social development [1,2]. In developed countries, various types of industrial robots have been widely used in many fields, such as the chemical industry, food processing, packaging, logistics, and machinery manufacturing. At present, industrial robots are an irreplaceable production means and an indispensable automation industrial equipment for modern enterprises, and they have begun to change people's lifestyle and industrial production model of mankind $[3,4]$.

In China, industrial robots are used in some industries, such as automobiles and electronics. The production cost of automation equipment is still relatively high for high-tech industrial robots in large state-owned enterprises, private enterprises, and SMEs (Small and Medium-sized Enterprises). Presently, more and more enterprises are getting aware of the advantage of automation industrial equipment, including the reduced labour costs, higher automation rate, and better production efficiency $[5,6]$. The industrial robot substituting for traditional manual operation develops noticeably, and it spread rapidly in different industries, getting more attention from various enterprises. Surely, it will be an important part of the robot industry market [7].

The research of industrial robots focuses on how to effectively control them. PLC (Programmable logic controller) is a control centre with the CPU (Central Processing Unit) as the core component and combines a series of cutting-edge technologies, such as modern computer technology and intelligent control system. It has many advantages, including comprehensive functions, low failure rate, convenient debugging, modular combination, and low energy consumption, and is a commonly used control centre in robot control systems at present [8]. The PLC controlled robot system has advantages, such as small size, high reliability, low failure rate, and high action accuracy, but its research cost is high and cannot be widely used [9]. PID control is a mature and reliable classical control strategy with a simple principle, high control accuracy, and wide application, yet a more accurate mathematical model is needed for object control [10]. The accuracy of the fuzzy control algorithm is not high, so it can be used in the application platform where the mathematical model is difficult to obtain or the dynamic characteristics are difficult to control. Moreover, it is 
easy to understand and has a strong antiinterference ability [11]. However, the fuzzy rules are based on expert knowledge or the experience of relevant personnel, which is difficult to adjust. Variable structure sliding mode control is an important method for the uncertain dynamic model system [12]. In a subspace state space with variations and disturbances, variable structure control systems have complete or high robustness, so it is more suitable for the industrial robot with multiple control solutions. Meanwhile, the variable structure control model leads to oscillation of industrial automation, so it has very limited application [13].

To sum up, the fuzzy controller and the traditional PID controller are innovatively integrated, and the fuzzy PID controller is constructed. Then, its calculation process is analysed, and its stability is evaluated through simulation experiments. Subsequently, the Mechatronics control system of an industrial robot is proposed, and the system is evaluated through experiment, providing an experimental basis for improvement of industrial robot efficiency.

\section{Construction of Mechatronics Control System for Industrial Robots based on Fuzzy PID \\ 2.1 Fuzzy PID Control Principle}

The control principle of fuzzy PID (Proportion Integral Differential) is to combine fuzzy logic with traditional PID technology [14]. There are three ways to combine fuzzy logic with PID. The first is fuzzy PID switching control. If the error category is large, fuzzy control is adopted. If the error category is small, PID control is adopted. The second is the hybrid fuzzy PID control, which combines the fuzzy controller with the PID controller in parallel to form a new control mode. The third is adaptive fuzzy PID control. The three parameters of the PID controller are corrected in real-time according to the fuzzy relationship between the three parameters of the PID controller, error change value, and the way of fuzzy reasoning [15].

The core advantage of the fuzzy logic controller is that it can be applied to nonlinear systems that are difficult to obtain mathematical models [16]. Moreover, the controller can be designed to apply heuristic rules reflecting human expert experience. The membership function of the relevant input/output language changes is generally determined in advance according to the nonlinear characteristics of the system. The traditional PID controller provides high sensitivity and tends to improve the stability of the overall feedback control system. Besides, the PID controller can reduce overshoot and allow greater gain through an added damping system. With differential, interference can be reduced, and the required set size can be maintained [17]. It is essential to design the structure and parameters to establish the fuzzy logic control of the fuzzy PID controller. The layout of this structure can establish the architecture of the controller, the input/output variables of the controller, and the format and number of fuzzy control rules. To design the PID of fuzzy PID controller successfully, it is necessary to select the optimal input and output proportional factor, which can enlarge or reduce the whole theoretical area. The input/output proportional factor can have a global influence on the control performance and robustness, so it is vital for the fuzzy PID controller adjustment and optimization.

In PID control, for different deviations e(U) and deviation change $\triangle \mathrm{e}(\mathrm{U})$, amplification coefficient $\left(U_{p}\right)$, integral amplification coefficient $\left(U_{i}\right)$, and differential amplification coefficient $\left(U_{d}\right)$ are different. If the value of $|\mathrm{e}(\mathrm{U})|$ is very large, the error should be eliminated as soon as possible, and the response time should be improved. At this time, the value of $U_{p}$ is relatively large, and to prevent overshoot, the values of $U_{d}$ and $U_{i}$ should be set to zero. If the value of $|e(U)|$ is small, to avoid the oscillation caused by overshoot and eliminate the error, the value of $U_{i}$ should be minimized, and the value of $U_{d}$ should be reduced, and the value of $U_{p}$ is determined by $|\triangle e(U)|$. If $|e(U)|=|\triangle e(U)|$, the controlled variable is deviating from the set value, and if both symbols are identical, the controlled variable is approaching the set value. When the controlled quantity gradually approaches the set value, the logarithmic proportional release can suppress the integral, so that overshoot and oscillation caused by the integral can be prevented. By comparison, if the controlled variable deviates from the set value for a long time, the whole system will be controlled slowly because the symbols of the two links of proportion and integral are opposite. Here, an adaptive fuzzy PID controller is used. The deviation e (U) and the deviation change $\triangle \mathrm{e}(\mathrm{U})$ are used as input values to adapt to the self-tuning requirements of PID parameters at different time points $e(U)$ and $\triangle e(U)$. The fuzzy control rules can correct the PID parameters in real-time, that is, to find the fuzzy relationship between the three parameters and $\mathrm{e}(\mathrm{U})$ and $\triangle \mathrm{e}(\mathrm{U})$.

\subsection{Fuzzy PID Controller Design}

The PID control method and fuzzy control technology are analysed in this chapter, and the fuzzy PID control technology is obtained based on the advantages of the two. The fuzzy PID control method is more flexible, applicable, and accurate. The integration of fuzzy control and PID control has been widely used in the industry.

Firstly, the structure of the fuzzy PID controller is explained. The fuzzy PID controller is divided into 
two parts: PID control and fuzzy control module. Here, the method of fuzzy adaptive PID parameters is adopted [18] to form an adaptive fuzzy PID controller. The motion of industrial robots can be decomposed into three directions, and three adaptive fuzzy PID controllers can control the motion of robots in these three directions. Then, how to construct an adaptive PID controller to control the longitudinal motion of the robot is described in detail. The deviation $e$ denotes the change of input in the controller, and the fuzzy relationship between deviation e and the changing PID controller should be obtained. Meanwhile, the changing system should be continuously detected and measured throughout the production process, and the fuzzy controller can adjust the relevant parameters of the PID controller for optimal system response, as shown in figure 1.

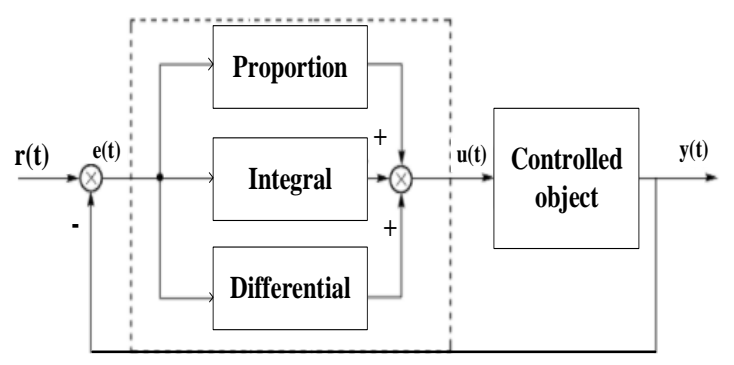

Figure 1: Structure of fuzzy PID controller
Secondly, fuzzy control rules are introduced [19]. Fuzzy controllers can infer the three parameters of PID through fuzzy rules and adjust the parameters according to the fuzzy matrix table [20]. The amplification factor $\left(U_{\mathrm{p}}\right)$, integral amplification factor $\left(U_{i}\right)$, and differential amplification factor $\left(U_{d}\right)$ should all be adjusted.

First, $\mathrm{U}_{\mathrm{p}}$ adjusting principle: if the deviation e is too large, $\Delta U_{p}$ is positive, namely, $U_{p}$ increases. If $e$ increases negatively, the system is in the overshoot state, and then the value of $U_{p}$ will be reduced. If the deviation e exceeds zero, and the deviation change value $(\triangle \mathrm{e})$ is negative, the system overshoot will gradually increase, and the $U_{p}$ should be reduced. If the deviation is positive, $\Delta U_{p}$ is positive to reduce the deviation. Language variables after fuzzifying input are set to be PL (Positive Large), PM (Positive Medium), PS (Positive Small), zero, NS (Negative Small), NM (Negative Medium), and NL (Negative Large). Second, the $U_{i}$ adjusting rule uses the integral separation principle [21], which is similar to the $U_{p}$ adjusting principle and can prevent integral saturation that causes overshoot. If the $U_{p}$ value increases, the $U_{i}$ value will be reduced accordingly. Third, the adjusting rule of $U_{d}$ is introduced. If the value of e is positive, it may lead to differential spill over when $U_{p}$ increases. Therefore, when the value of $U_{p}$ is large, the value of $U_{d}$ should be small. The fuzzy rule tables for the three parameters are shown in tables 1,2 , and 3 .

Table $1 U_{p}$ fuzzy rule table

\begin{tabular}{|c|c|c|c|c|c|c|c|c|}
\hline & \multicolumn{8}{|c|}{$\triangle \mathrm{e}$} \\
\hline \multirow{8}{*}{ e } & $\Delta U_{p}$ & NL & NM & NS & 0 & PS & $\mathrm{PM}$ & $\mathrm{PL}$ \\
\hline & NL & NL & NL & NM & NM & NS & 0 & 0 \\
\hline & NM & NL & NL & NM & NM & NS & 0 & 0 \\
\hline & NS & NM & NM & NM & NS & 0 & PS & PM \\
\hline & 0 & NM & NS & NS & 0 & PS & PM & PM \\
\hline & PS & NS & NS & 0 & PS & PS & PM & PM \\
\hline & PM & 0 & 0 & PS & PM & PM & PM & PL \\
\hline & PL & 0 & 0 & PS & PM & PM & PS & PS \\
\hline
\end{tabular}

Table 2 U fuzzy rule table

\begin{tabular}{ccccccccc}
\hline & & & \multicolumn{1}{c}{$\Delta \mathrm{e}$} \\
\hline & $\triangle \mathrm{U}_{\mathrm{i}}$ & $\mathrm{NL}$ & $\mathrm{NM}$ & $\mathrm{NS}$ & 0 & $\mathrm{PS}$ & $\mathrm{PM}$ & $\mathrm{PL}$ \\
& $\mathrm{NL}$ & $\mathrm{NM}$ & $\mathrm{NM}$ & $\mathrm{NS}$ & $\mathrm{NS}$ & 0 & 0 & 0 \\
$\mathrm{NM}$ & $\mathrm{NM}$ & $\mathrm{NM}$ & $\mathrm{NS}$ & $\mathrm{NS}$ & 0 & 0 & 0 \\
$\mathrm{e}$ & $\mathrm{NS}$ & $\mathrm{NS}$ & $\mathrm{NS}$ & 0 & 0 & 0 & PS \\
& $\mathrm{NS}$ & $\mathrm{NS}$ & 0 & 0 & 0 & 0 & PS & PS \\
& 0 & 0 & 0 & 0 & 0 & PS & PS \\
& PS & 0 & 0 & 0 & PS & PS & PS & PM \\
& PM & 0 & 0 & 0 & PS & PS & PM & PM \\
\hline
\end{tabular}


Table $3 U_{d}$ fuzzy rule table

\begin{tabular}{|c|c|c|c|c|c|c|c|c|}
\hline & & \multicolumn{7}{|c|}{$\triangle \mathrm{e}$} \\
\hline \multirow{8}{*}{$\mathrm{e}$} & $\Delta \mathrm{U}_{\mathrm{d}}$ & NL & NM & NS & 0 & PS & PM & PL \\
\hline & NL & PL & PL & PM & PM & PS & PS & 0 \\
\hline & NM & PL & PL & PM & PM & PS & 0 & 0 \\
\hline & NS & PM & PM & PM & PS & 0 & NS & $\mathrm{NM}$ \\
\hline & 0 & PM & PS & PS & 0 & NS & NM & $\mathrm{NM}$ \\
\hline & PS & PS & PS & 0 & PS & PS & PM & $\mathrm{PM}$ \\
\hline & PM & 0 & 0 & NS & NM & NM & NM & NL \\
\hline & PL & 0 & NS & NS & NM & NM & NL & NL \\
\hline
\end{tabular}

The results of fuzzy reasoning cannot be used until the parameters are modified, so it is necessary to fuzzy the results of fuzzy reasoning. The centre of gravity method [22] can fuzzy the results and obtain the accurate distribution of the control quantity. Thus, the membership of each fuzzy subset can be obtained.

According to the fuzzy control model of each parameter and the fuzzy matrix table, the modified parameters are found out and replaced through the integral separation PID control algorithm for the final adjusting parameters. The algorithm can be expressed as below.

$$
\begin{aligned}
\mathrm{f}(U)= & U_{\mathrm{p}} \operatorname{error}(U)+\alpha u_{i} \sum_{j=0}^{U} \operatorname{error}(j) T \\
& +U_{P}(\operatorname{error}(U)-\operatorname{error}(U-1)) / T
\end{aligned}
$$

In equation (1), $T$ represents the sampling period, $\alpha$ denotes the switching coefficient of the integral term. Then, the final value of adjusting parameters can be expressed as below.

$$
\begin{aligned}
& U_{P}=U_{P 0}+\Delta U_{P} \\
& U_{\mathrm{i}}=U_{i 0}+\Delta U_{i} \\
& U_{d}=U_{d 0}+\Delta U_{d}
\end{aligned}
$$

In equation (2), $U_{\mathrm{P} 0}, \mathrm{U}_{\mathrm{i} 0}$, and $\mathrm{U}_{\mathrm{d} 0}$ stand for the corresponding initial values.

Finally, the calculation process of the fuzzy PID control algorithm is presented. The fuzzy PID controller can find PID controller proportion, integral, and differential coefficient through fuzzy controller [23] for the best system state. During system operation, the fuzzy reasoning results are analysed, searched, and calculated to realize the realtime self-adjusting of the three parameters of the PID controller [24]. Consequently, the appropriate parameter values are obtained for the best system response. The flowchart of the algorithm is shown in figure 2 .

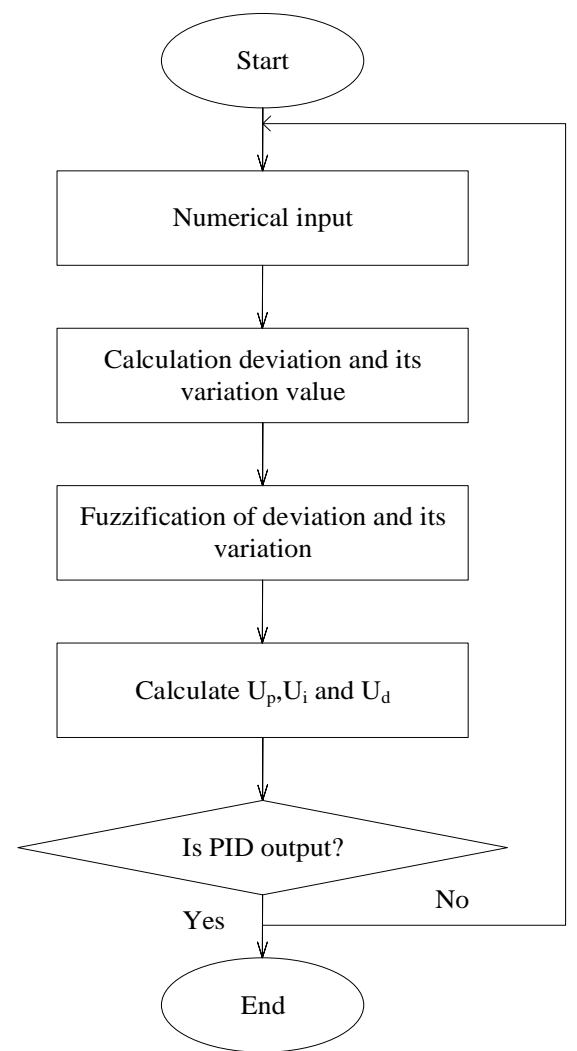

Figure 2: Adaptive fuzzy PID algorithm

\subsection{Construction of Mechatronics Control System for Industrial Robot}

The mechatronics control system can automatically control key parameters in production through mechatronics control equipment. When the equipment deviates from the normal state due to external interference, it can automatically be adjusted to the required numerical range for production. During production, technologies and process conditions change constantly. Especially, in continuous chemical production, where all kinds of equipment are interrelated. When the process conditions of an equipment change, some parameters of other equipment may fluctuate, and thus deviating from the normal process conditions. Automatic adjustment indicates that there is no need for manual interference, and the mechatronics control system is composed of the controllers and the controlled objects. 
For complex control tasks, a typical mechatronics control system should also include an adjusting component, execution component, measurement transmission component, and comparison component. The components of the mechatronics control system of industrial robots are very complex.
Here, the industrial transport robot is chosen for the design of the Mechatronics control system of the industrial transport robot. The system is mainly composed of three parts: the transport robot, the console, and the mechatronics control software [25]. Figure 3 shows the overall system design.

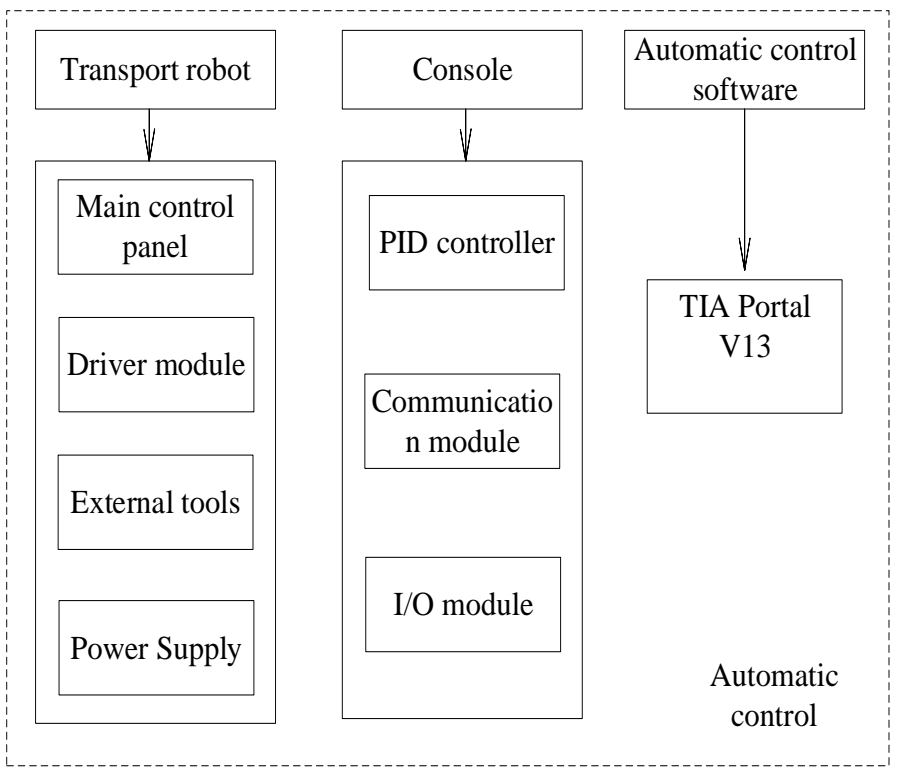

Figure 3: Structure of mechatronics control system

The functions of each component of the system are shown in table 4 .

Table 4 Function of the component of mechatronics control system

\begin{tabular}{cc}
\hline Components & Functions \\
\hline Main control panel & Information analysis and processing \\
Driver module & Robot trajectory control \\
Communication module & Data transmission \\
External tools & Workpiece grasping \\
Power supply & Energy suppling \\
PID controller & Robot motion control \\
I/O module & Data collection \\
TIA Portal V13 & Communication of control software for robot end system;
\end{tabular}
receive the operator's instructions and feedback; display and store sensor information

The control software of the transport robot must be improved and perfected.

Firstly, the software should communicate with the console software system. Secondly, the software should receive the commands sent by the console software system, perform accordingly, and feedback the execution results. Thirdly, it should control the movement of the robot. Fourthly, it must control the action of the workpiece grasping tool.

Finally, it should collect the action signal of the grasping tool and feed it back to the console.

Only the efficient cooperation of software and hardware can achieve the best performance of the system.

\section{Fuzzy PID Simulation and Mechatronics Control Results of Industrial Robots}

\subsection{Simulation Results of Fuzzy PID Controller}

The output response and motion response of the fuzzy PID controller of industrial transport robot are visually analysed, as shown in figure $4 \mathrm{~A}$ and figure 4B.

Figure 4A shows that the industrial transport robot responds quickly to the grasping operation, and the system begins to stabilize at 40 seconds. 


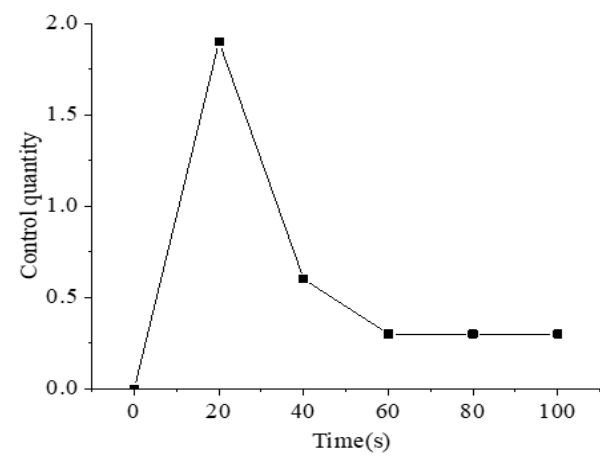

A

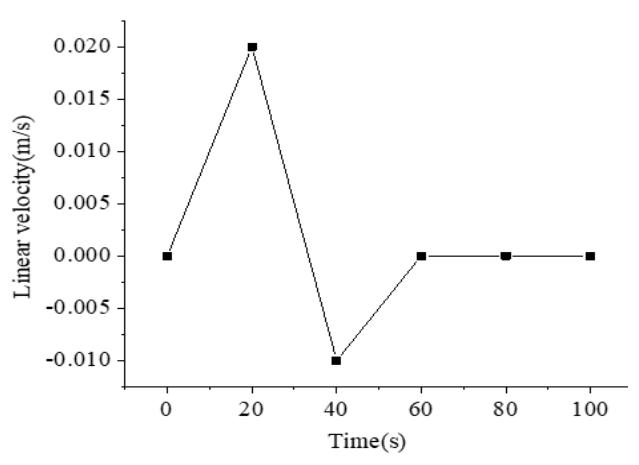

B

Figure 4: Output response and motion response of the fuzzy PID controller (A: simulation results of control quantity, B: simulation results of linear speed)

Figure 4B shows the speed response of the controller. When the system is stable, the speed approaches zero. The speed curve indicates that the speed fluctuates greatly, there is a large impact, and the system may have a certain overshoot. Besides, the system also has a small steady-state error.

Then, the fuzzy results of the PID controller are analysed, that is, the fuzzy results of PID controller deviation e are visualized, as shown in figure 5 .

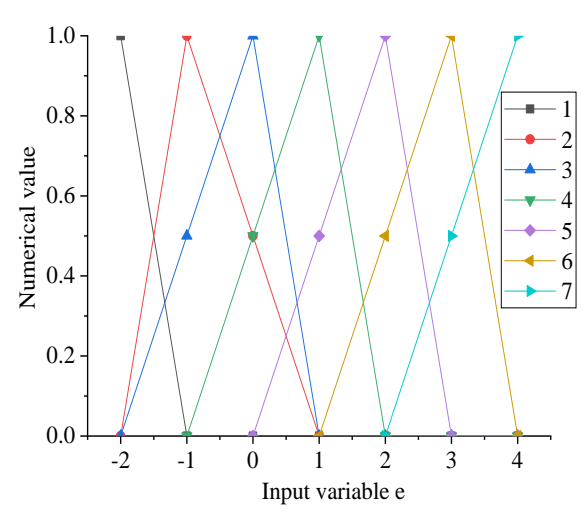

Figure 5: PID Fuzzy results of controller deviation (1: NL 2: NM 3: NS 4: zero 5: PS 6:PM 7: PL)

Figure 5 suggests that the fuzzy deviation e of the PID controller conforms to the standard design created by Mamdani.
The domain of the three parameters of the PID controller is between $[-2,4]$, which indicates that the fuzzy deviation e is completed.

\subsection{Experimental Comparison of Different Controllers}

Three kinds of controllers, namely integral separation PID controller, fuzzy controller, and fuzzy PID controller, are used in industrial transport robot operation experiments. The comparison results are shown in the figure.

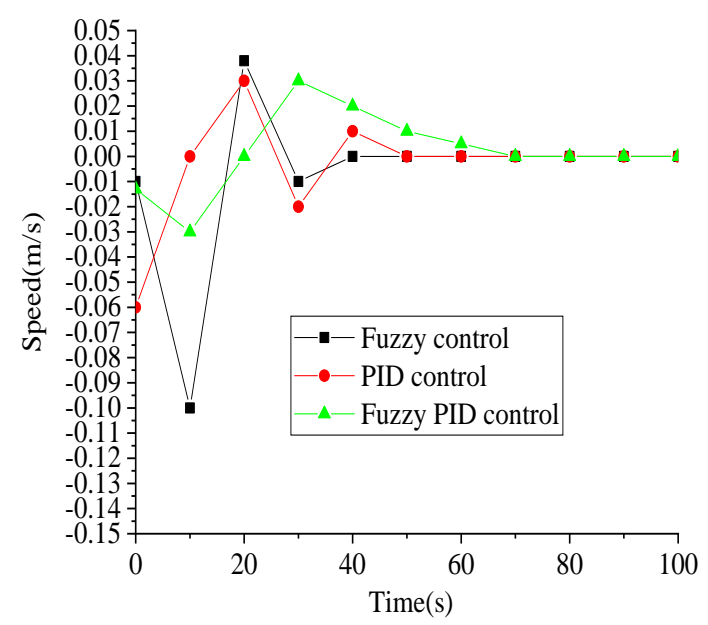

Figure 6: Operation of industrial transport robot under different controllers

Figure 6 implies that although the speed of the industrial transport robot approaches zero under the three controllers, and the system reaches stability, the response speed of the robot through fuzzy control algorithm is relatively fast, and it tends to balance quickly. However, the speed fluctuation is large, and the system is prone to overshoot. By comparison, although the integral separation PID controller can reduce the error, improve the control accuracy, and increase the response time, the system will also appear overshoot. The fuzzy PID controller is superior to the integral separation PID controller, and the controller has a fast speed response. Compared with the fuzzy controller, the speed fluctuation range is small, no overshoot occurs, and the control accuracy is high. It indicates that the effect of a fuzzy PID controller is the best.

\subsection{Experimental Results of the Industrial Transport Robot}

The system response and speed response of the industrial transport robot are visualized and analysed, as shown in figure 7A and figure 7B. 


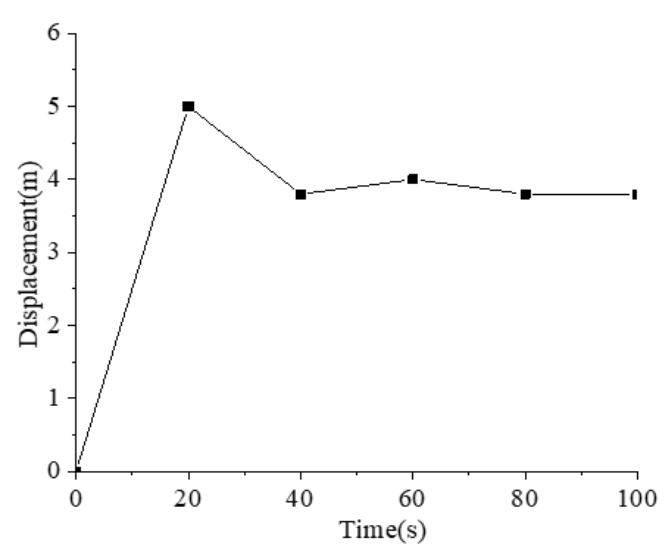

A

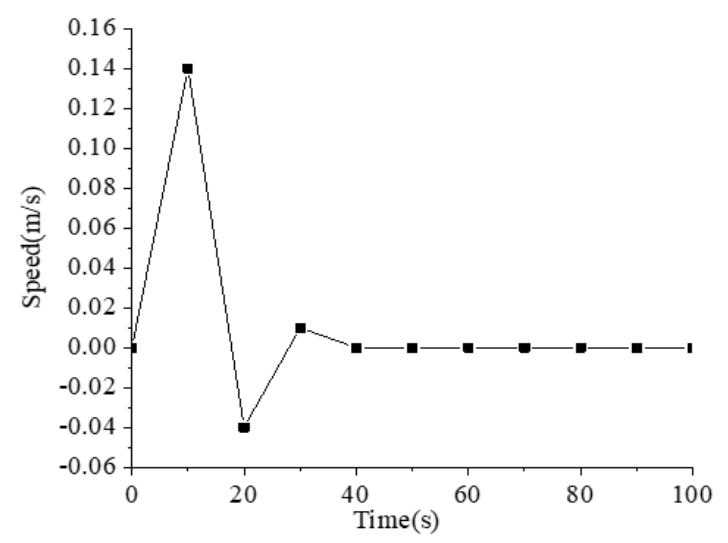

$\mathrm{B}$

Figure 7: Response of industrial transport robot (A: system response B: speed response)

Figure 7A that when the robot moves forward and backward, it does not stop moving when it reaches the set value of 3.5 meters. Then, it moves forward about 1.5 meters before it moves backward, approaches the set value of 3.5 meters, and then hovers at the position of 3.5 meters until the system reaches a stable state. Figure $7 \mathrm{~B}$ indicates that the oscillation of the industrial transport robot is not strong, and it gradually stabilizes after 40 seconds.

\subsection{Response Results of Industrial Transport Robot During Operation}

The system response and speed response are visually analysed of the industrial transport robot during operation. The results are shown in figure $8 \mathrm{~A}$ and figure 8B.

Figure 8A suggests that the response fluctuation is also relatively small, and the speed change within 10 seconds is between [-0.02, 0.01]. Moreover, the movement of the robot is very stable, and there is no significant oscillation. Figure $8 \mathrm{~B}$ implies that the response time of the system is short when the industrial transport robot is operating, and the robot moves around the set value of $3.5 \mathrm{~m}$.

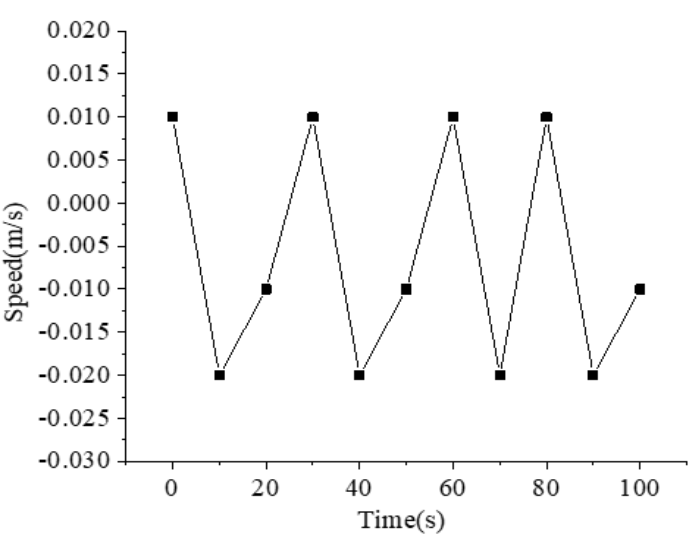

A

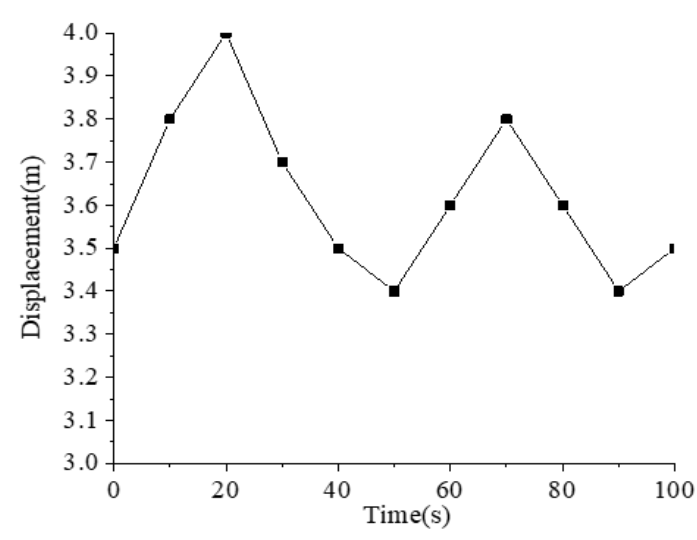

B

Figure 8: Response of industrial transport robot during operation (A: speed response B: system response)

\section{Conclusion}

The fuzzy PID controller is constructed through the integration of fuzzy logic and traditional PID technology. The constructed controller has advantages, such as flexibility, easy applicability, and high accuracy. Afterward, the calculation process of the fuzzy PID control algorithm is proposed, and the proportion, integral, and differential coefficients of the PID controller are calculated through the fuzzy controller for the best system state. The controller simulation and system experiment show that the response of the system is fast when the industrial transport robot is grasping workpieces, while the speed fluctuation is large, there is a large impact, and the system has an overshoot. Besides, the system also has a small steady-state error, but the fuzzification of PID deviation e conforms to the standardized design established by Mamdani. The performance of fuzzy PID controllers is better than that of fuzzy controllers and traditional PID controllers. The response time of the robot is short and stable when operating. 
There are still some shortcomings. First, that the controller will have a coupling effect when the robot moves in different directions, which will have a certain impact on the experimental results. Second, human-computer interaction is not considered in the system design. This will affect the operational intelligence of the robot, which is the focus of the next research.

\section{References}

[1] Tang G, Webb P, Thrower J. (2018) The development and evaluation of Robot Light Skin: A novel robot signalling system to improve communication in industrial human-robot collaboration. Robotics and Computer-Integrated Manufacturing, 56, 85-94.

[2] Chaudhary H, Panwar V, Prasad R, et al. (2016) Adaptive neuro fuzzy based hybrid force/position control for an industrial robot manipulator. Journal of Intelligent Manufacturing, 27(6), 1299-1308.

[3] Zhou Y M, Hohimer C, Proietti T, et al. (2021) Kinematics-based Control of an Inflatable Soft Wearable Robot for Assisting the Shoulder of Industrial Workers. IEEE Robotics and Automation Letters, PP (99), 1-1.

[4] Palpacelli M. (2016) Static performance improvement of an industrial robot by means of a cable-driven redundantly actuated system. Robotics and Computer-Integrated Manufacturing, 38(APR.), 1-8.

[5] Wang M, Feng C. (2019) Impacts of oriented technologies and economic factors on China's industrial climate mitigation. Journal of Cleaner Production, 233(OCT.1), 1016-1028.

[6] Yahaghi E, Movafeghi A. (2019) Contrast Enhancement of Industrial Radiography Images by Gabor Filtering with Automatic Noise Thresholding. Russian Journal of Nondestructive Testing, 55(1), 73-79.

[7] Yang K, Yang W, Cheng G, et al. (2018) A new methodology for joint stiffness identification of heavy duty industrial robots with the counterbalancing system. Robotics and Computer-Integrated Manufacturing, 53, 58-71.

[8] Yerden A U, Akkus N. (2020) Virtual Reality Remote Access Laboratory for Teaching Programmable Logic Controller Topics. International Journal of Engineering Education, 35(5), 1708-1721.

[9] Shinkaiouchi F, Koyama S, Ono Y, et al. (2016) Predictions of Cleavability of Calpain Proteolysis by Quantitative Structure-Activity Relationship
Analysis Using Newly Determined Cleavage Sites and Catalytic Efficiencies of an Oligopeptide Array. Molecular \& Cellular Proteomics, 15(4), 1262-1280.

[10] Trafczynski M, Markowski M, Alabrudzinski S, et al. (2016) The influence of fouling on the dynamic behavior of PID-controlled heat exchangers. Applied Thermal Engineering, 109, 727-738.

[11] Yang H, Wang H. (2016) Robust adaptive faulttolerant control for uncertain nonlinear system with unmodeled dynamics based on fuzzy approximation. Neurocomputing, 173 (JAN.15PT.3), 1660-1670.

[12] Wang N, Sun J C, Liu Y C. (2016) Direct adaptive self-structuring fuzzy control with interpretable fuzzy rules for a class of nonlinear uncertain systems. Neurocomputing, 173 (JAN.15PT.3), 1640-1645.

[13] Beluli V M. (2019) Smart beer production as a possibility for cyber-attack within the industrial process in automatic control. Procedia Computer Science, 158, 206-213.

[14] Khandekar A V, Chakraborty S. (2016) Application of fuzzy axiomatic design principles for selection of non-traditional machining processes. The International Journal of Advanced Manufacturing Technology, 83(1-4), 529-543.

[15] Saad, Babesse, Djameleddine, et al. (2016) Comparison between RST and PID controllers performance of a reduced order model and the original model of a hydraulic actuator dedicated to a semi-active suspension [J]. Military operations research, 13(1), 66-71.

[16] Mustafa G I Y, Wang H P, Tian Y. (2019) Vibration control of an active vehicle suspension systems using optimized model-free fuzzy logic controller based on time delay estimation. Advances in Engineering Software, 127(JAN.), 141-149.

[17] Lapa K, Cpalka K. (2017) Flexible fuzzy PID controller (FFPIDC) and a nature-inspired method for its construction. IEEE Transactions on Industrial Informatics, PP (99), 1-1.

[18] Azeloglu C 0, Sagirli A, Edincliler A. (2016) Vibration mitigation of nonlinear crane system against earthquake excitations with the selftuning fuzzy logic PID controller. Nonlinear Dynamics, 84(4), 1915-1928.

[19] Wang N, Sun J C, Liu Y C. (2016) Direct adaptive self-structuring fuzzy control with interpretable fuzzy rules for a class of nonlinear uncertain 
systems. Neurocomputing, 173(JAN.15PT.3), 1640-1645.

[20] Elsayed A A A, Malkawi G, Ahmad N. (2020) On the solution of fully fuzzy Sylvester matrix equation with trapezoidal fuzzy numbers. Computational and Applied Mathematics, 39(4), 1-22.

[21] Tran D, Yucelen T, Sarsilmaz S B. (2021) Finitetime control of multiagent networks as systems with time transformation and separation principle. Control Engineering Practice, 108(18), 104717.

[22] Wieczorek B, Górecki, Jan, Kukla M, et al. (2017) The Analytical Method of Determining the Center of Gravity of a Person Propelling a Manual Wheelchair. Procedia Engineering, 177(Complete), 405-410.
[23] Wei G, Shen J. (2018) Asymptotic behavior of impulsive neutral delay differential equations with positive and negative coefficients of Euler form. Advances in Difference Equations, 44(1112), 1089-1096.

[24] Kumar M S, Mahadevan K. (2016) Paper Machine Industrial Analysis on Moisture Control Using BF-PSO Algorithm and Real Time Implementation Setup through Embedded Controller. Journal of Electrical Engineering and Technology, 11(2), 490-498.

[25] Lungu M, Lungu R. (2016) Automatic control of aircraft lateral-directional motion during landing using neural networks and radio-technical subsystems. Neurocomputing, 171(JAN.1), 471481. 